\title{
Lattice Boltzmann simulation of fluid flow in fracture networks with rough, self-affine surfaces
}

\author{
Mahyar Madadi ${ }^{1,2}$ and Muhammad Sahimi ${ }^{1,3}$ \\ ${ }^{1}$ Institute for Advanced Studies in Basic Science, Gava Zang, Zanjan 45195-159, Iran \\ ${ }^{2}$ Institute for Computer Applications 1, University of Stuttgart, Pfaffenwaldring 27, D-70569 Stuttgart, Germany \\ ${ }^{3}$ Department of Chemical Engineering, University of Southern California, Los Angeles, California 90089-1211
}

(Received 12 March 2002; revised manuscript received 3 September 2002; published 14 February 2003)

\begin{abstract}
Using the lattice Boltzmann method, we study fluid flow in a two-dimensional (2D) model of fracture network of rock. Each fracture in a square network is represented by a $2 \mathrm{D}$ channel with rough, self-affine internal surfaces. Various parameters of the model, such as the connectivity and the apertures of the fractures, the roughness profile of their surface, as well as the Reynolds number for flow of the fluid, are systematically varied in order to assess their effect on the effective permeability of the fracture network. The distribution of the fractures' apertures is approximated well by a log-normal distribution, which is consistent with experimental data. Due to the roughness of the fractures' surfaces, and the finite size of the networks that can be used in the simulations, the fracture network is anisotropic. The anisotropy increases as the connectivity of the network decreases and approaches the percolation threshold. The effective permeability $K$ of the network follows the power law $K \sim\langle\delta\rangle^{\beta}$, where $\langle\delta\rangle$ is the average aperture of the fractures in the network and the exponent $\beta$ may depend on the roughness exponent. A crossover from linear to nonlinear flow regime is obtained at a Reynolds number $\operatorname{Re} \sim O(1)$, but the precise numerical value of the crossover Re depends on the roughness of the fractures' surfaces.
\end{abstract}

DOI: 10.1103/PhysRevE.67.026309

PACS number(s): 47.55.Mh, 47.11.+j, 02.50.-r, 62.20.Mk

\section{INTRODUCTION}

Flow and transport in systems with rough surfaces and boundaries are relevant to a wide variety of scientific and industrial problems. For example, natural porous media and rock contain a wide variety of pores and fractures with broad distributions of sizes and shapes (for recent reviews see, for example, Sahimi [1]). Transport of fluids in such porous media involves not only flow through the microscopic pores, but also through the fractures which are typically much larger than the pores. Most rock masses contain complex and interconnected fracture networks, the presence of which is critical to the economics of oil recovery from underground reservoirs, generation of steam from geothermal reservoirs for use in power plants, and the development of groundwater resources, as the fractures provide high permeability paths for fluid flow and transport processes.

Flow in unfractured porous media is now relatively well understood [1]. One typically represents the pore space by a network of interconnected pores with distributed sizes and connectivities, and utilizes either computer simulations, or analytical approximations, such as the effective-medium approximation, or the renormalization group theory, in order to compute the effective properties of the network, such as its permeability and its electrical conductivity. However, the same level of understanding has not yet been obtained for fluid flow through a network of fractures. In the early work on modeling of flow through fractures, the fractures were typically represented by channels between two parallel flat plates of length $l$ for which the volumetric flow rate $q$ is given by

$$
q=\frac{w \delta^{3} \Delta P}{12 \eta l}
$$

where $w$ is the width of the fracture and $\delta$ its aperture, $\Delta P / l$ is the pressure gradient along fracture, and $\eta$ is the viscosity of the fluid. Thus according to this model $q$ depends on the third power of $\delta$, and the effective permeability $K$ of the fracture varies as $\delta^{2}$. The problem of fluid flow through a fracture network is then reduced to one in a network of such channels.

However, there is now ample experimental evidence [2-6] that the internal surface of natural fractures in rock masses is very rough, and that the roughness follows selfaffine fractal statistics. More specifically, consider the internal surface of a fracture with a height $h(x, y)$ which is a single-valued function. The surface is assumed not to have any overhangs, and the coordinates $(x, y)$ lie in the mean plane of the fracture. Self-affinity of the rough surface implies that it exhibits scale invariance under rescaling except that, unlike self-similar structures, one has directiondependent rescaling factors such that, $x \rightarrow \lambda_{x} x, y \rightarrow \lambda_{y} y$, and $h \rightarrow \lambda_{h} h$. Typically, isotropy in the mean plane of the fracture can be assumed. As a result, one may assume that $\lambda_{x}=\lambda_{y}$ $=\lambda$ and $\lambda_{h}=\lambda^{H}$, so that

$$
h(x, y)=\lambda^{-H} h(\lambda x, \lambda y),
$$

where $H$ is the roughness or Hurst exponent.

For fracture surface of materials a more or less universal value of the roughness exponent, $H \simeq 0.8$, has been reported by several research groups (for a recent review see, for example, Sahimi [7]). As for the internal surface of fracture of rock, which is the subject of this paper, an estimate $H$ $\simeq 0.85$ was reported by Schmittbuhl et al. [4] for granitic faults, very close to the universal value for fracture surface of materials. However, Cox and Wang [5], Odling [5], and others [6] analyzed extensive data for a variety of rock joints and reported nonuniversal values of the roughness exponent 
in the range $0<H \leqslant 0.85$, and therefore in this paper we consider roughness exponents in the interval $(0,1)$. There is also considerable experimental evidence for deviations from the cubic law, Eq. (1), $q \sim \delta^{3}$, which have been attributed [8] to the roughness of the internal surface of fracture. In nature, of course, fractures possess self-affine surfaces over a range of length scales limited by a lower and an upper cutoff length, where the size of the fracture itself is the obvious upper cutoff, while the self-affinity may break down below some characteristic microscopic length, such as the typical size of the grains that constitute the rock.

The roughness of the internal surface of fractures has a practical implication in that, it makes it clear that the task of simulation of fluid flow in fractures with a realistic model for the roughness of their internal surface is very complex. Since the problem of fluid flow in a single fracture with rough, self-affine internal surface cannot be solved analytically, one must resort to numerical simulations. The numerical methods that have been used so far are mostly of two types. (i) In one approach the simulations are based on discretizing the governing equations-the usual continuity and the Stokes' equations - by a finite-difference (FD) or finite-element (FE) method and solving the resulting set of equations [9]. However, if the effect of surface roughness of the fracture is to be taken into account, the FD or FE grid must be very refined near the surface, which would then require prohibitive computations. (ii) Alternatively, and much more efficiently, one may use a lattice gas (LG) or lattice Boltzmann (LB) method [10] which are ideally suited for simulation of fluid flow in systems that have very irregular geometries. To our knowledge, Gutfraind and Hansen [11] and Zhang et al. [12] were the first to use a LG method to study fluid flow in a single fracture with self-affine internal surfaces. More recently, Drazer and Koplik [13] used the LB method to study fluid flow in a single fracture with self-affine internal surface. For $H$ close to 1 , they also derived an analytical approximation that relates the fracture's effective permeability to its effective aperture, the roughness exponent $H$, and other relevant parameters. Madadi et al. [13] and Van Siclen [13] carried out a similar study in which, in addition to fluid flow, conduction in a single fracture (i.e., conduction in a single fracture saturated by a conducting fluid, such as brine) was also studied, and various analytical approximations for the flow and conduction properties were examined.

The fracture pattern in rock masses is, however, typically very branched and appears as a highly interconnected network [14]. In fact, analysis of Chelidze and Gueguen [15] indicated that the three-dimensional (3D) fracture network of rock masses may even be a fractal object with a fractal dimension $D_{f} \simeq 2.5$, and may have a structure close to that of the sample-spanning percolation cluster at the percolation threshold $p_{c}[16,17]$ which also has a fractal dimension of about 2.5. Therefore a complete understanding of fluid flow in a fracture network requires, at the very least, a model with two key ingradients: (i) Each fracture must have rough, selfaffine internal surface, consistent with the experimental evidence described above, and (ii) such fractures must be interconnected, forming a macroscopic network, similar to fracture networks that typically exist in rock masses. Al- though several models of fracture networks have been proposed in the past [18-24], almost all of them are essentially equivalent to random resistor networks that have been used in the past for modeling percolation and conduction in disordered materials, or fluid flow in unfractured porous media. A notable exception is the work of Adler and co-workers $[25,26]$. In their original work [25], the network was made of plane polygonal fractures that were randomly distributed in space. The effective permeability of the system was then computed by numerically solving the flow equations. In subsequent work [26], the roughness of the fracture surfaces was taken into account and percolation and conduction properties of the system were studied.

In this paper, we propose a different model of fracture network of rock that contains the two key ingradients mentioned above. In our model, each fracture possesses selfaffine internal surfaces. As the first step toward a full, 3D model, we use the LB method to study in this paper fluid flow in a square network of such fractures. We also vary the connectivity of the network in order to assess its effect on the flow properties. We have also studied dispersion and miscible displacements in this model, the results of which will be reported in a future paper. Work on a 3D model of interconnected $3 \mathrm{D}$ fractures is also in progress.

This paper is organized as follows. In the next section, we describe briefly the fracture network model, after which we discuss the LB method that we employ to carry out the fluid flow simulations. We then present and discuss the results. The paper is summarized in the last section.

\section{GENERATION OF THE FRACTURE NETWORK}

Each fracture is initially represented by a $2 \mathrm{D}$ channel between two parallel flat surfaces. We then generate the roughness profile and superimpose it on top of the flat surfaces. To generate the roughness profile, we use a 1D fractional Brownian motion (FBM). Briefly, the FBM is a stochastic process $B_{H}(r)$ with the properties that $\left\langle B_{H}(\mathbf{r})-B_{H}\left(\mathbf{r}_{0}\right)\right\rangle$ $=0$, and

$$
\left\langle\left[B_{H}(\mathbf{r})-B_{H}\left(\mathbf{r}_{0}\right)\right]^{2}\right\rangle \sim\left|\mathbf{r}-\mathbf{r}_{0}\right|^{2 H},
$$

where $\mathbf{r}=(x, y, z)$ and $\mathbf{r}_{0}=\left(x_{0}, y_{0}, z_{0}\right)$ are two arbitrary points in space, and $H$ is the Hurst exponent. The $d$-dimensional surfaces that are generated by the FBM have exactly the same scaling property as in Eq. (2) with the same Hurst or roughness exponent. For $H>\frac{1}{2}$ the FBM displays persistence or positive correlations, i.e., a large or small height at $\mathbf{r}$ is likely to be followed by a similar trend at $\mathbf{r}$ $+\Delta \mathbf{r}$, whereas for $H<\frac{1}{2}$ the FBM generates antipersistence or negative correlations, i.e., a large or small height at $\mathbf{r}$ is likely to be followed by its opposite at $\mathbf{r}+\Delta \mathbf{r}$. To generate the 1D FBM profile, a fast Fourier transformation method and the power spectrum representation of the FBM are used. The power spectrum $S(\omega)$ of a 1D FBM is given by

$$
S(\omega)=\frac{a_{0}}{\omega^{2 H+1}},
$$



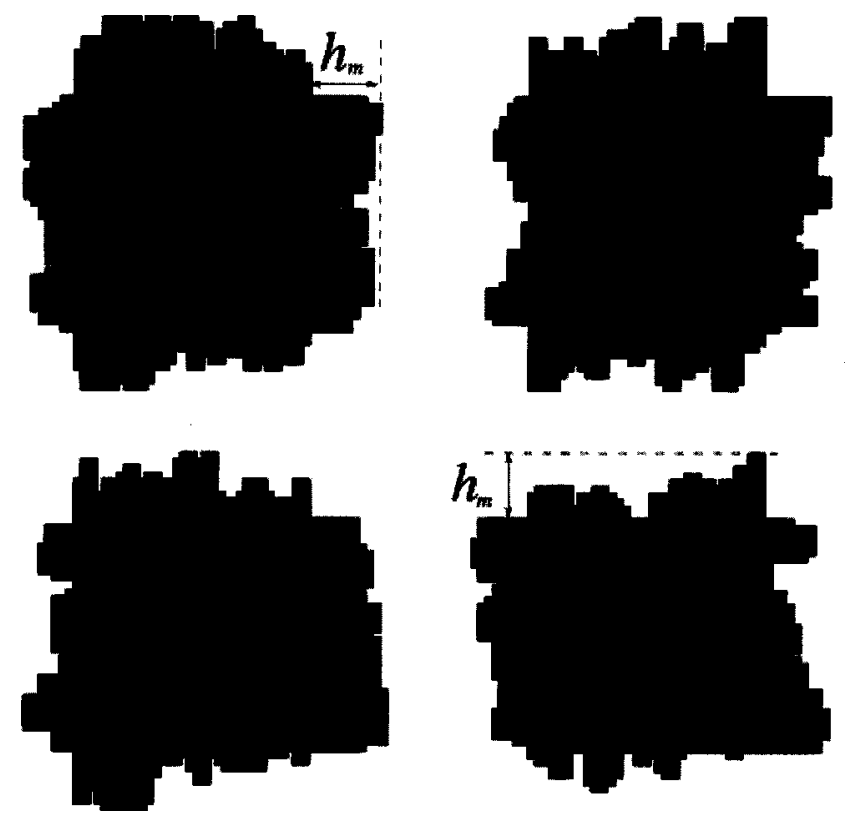

FIG. 1. A small portion of the model of the fracture network with rough internal surfaces. $h_{m}$ indicates the maximum height of the surface profile.

where $\omega$ is the frequency, and $a_{0}$ is a constant. Using this method, we generate self-affine surfaces of root mean square thickness $\ell$. The local apertures vary with position within the fractures. Each fracture is then assigned an effective aperture $\delta$, defined as the distance between the two mean surfaces parallel to the flow direction: $\delta=L_{y}-2 \ell$, where $L_{y}$ is the linear size of the fracture transverse to the flow direction.

All the fractures are assumed to have the same length. As shown in Fig. 1, each fracture is characterized by a quantity $h_{m}$, representing the maximum height of its rough surface. The motivation for defining a maximum height $h_{m}$ is to have a single, well-defined parameter with which a fracture network, with a fixed roughness exponent $H$, can be characterized. While, in practice, the fractures in a network may not all have the same maximum height $h_{m}$, allowing $h_{m}$ to vary among the fractures would generate a network that is difficult to characterize with a physical parameter. On the other hand, varying $h_{m}$ (that is, translating the upper and lower surfaces normal to the mean plane) allows us to vary the distance between the upper and lower surfaces and obtain fractures with a range of apertures. We have used several values of $h_{m}$ in order to study the effect of the fractures' apertures on the macroscopic properties of the fracture network. In particular, we have used $h_{m}>\frac{1}{2} L_{y}$, where $L_{y}$ is the linear extent of the fractures transverse to the flow direction. Such values of $h_{m}$ result in overlap of the top and bottom surfaces of a fracture, leading to its effective closure. Thus this method generates, in a natural way, the topology of a percolation cluster for the fracture network.

The fractures that are generated in this way are then arranged as a square network. As shown in Fig. 1, the intersections of the fractures are simple squares, obtained as the intersection of the channels without any surface roughness. The space in the fractures' intersections are also discretized finely for simulation of fluid flow (see below). In addition to generating fracture with $h_{m}>\frac{1}{2} L_{y}$, which may result in the closure of some fractures, we have also removed, as a simple way of varying the connectivity of the fracture network, a randomly selected fraction of the fractures. That is, we generate a percolation network in which each intact bond is a fracture with internal self-affine surfaces. The motivation for using this algorithm is to make the model consistent with the results of Chelidze and Gueguen [15], who analyzed the topology of rock fracture networks based solely on their connectivity (without any regard for the aperture or flow properties of the individual fractures), and proposed that the topology of such networks is similar to that of a samplespanning percolation cluster at or very near the percolation threshold.

Various network sizes up to $150 \times 150$ have been used. We have also generated up to 20 realizations of the networks, and most of the results that are presented below represent the averages over all the realizations. To our knowledge, these calculations represent some of the most intensive computational modeling of fluid flow in a system with a nontrivial geometry, using the LB method.

\section{LATTICE BOLTZMANN SIMULATION}

In the LB method, fictitious particles move on the site of a lattice which then collide with each other and scatter to the neighboring sites. Various collision rules have been proposed in the past [10]. We use the nine-velocity LB method [10] for simulating fluid flow in the fracture network. The open space of each fracture, in which the fluid flows, is discretized into a $32 \times 32$ lattice, where the lattice spacing is $\Delta x$. Such a mesh proved to provide sufficient resolution for the computations. For each lattice point $\mathbf{x}$ we define, at time $t$, a distribution function $f\left(\mathbf{x}, \mathbf{v}_{\alpha}, t\right)$, where the velocity $\mathbf{v}_{\alpha}$ can take on any of the nine values [10]. The distribution function $f_{\alpha}(\mathbf{x}, t)=f\left(\mathbf{x}, \mathbf{v}_{\alpha}, t\right)$ satisfies the discrete Boltzmann equation:

$$
f_{\alpha}\left(\mathbf{x}+\mathbf{v}_{\alpha} \Delta t, t+\Delta t\right)-f_{\alpha}(\mathbf{x}, t)=-\frac{\Delta t}{\tau}\left[f_{\alpha}(\mathbf{x}, t)-f_{\alpha}^{\mathrm{eq}}(\mathbf{x}, t)\right]
$$

where $\Delta t$ is the time step, $\tau$ is a relaxation time, and $f_{\alpha}^{\mathrm{eq}}(\mathbf{x}, t)$ is the quasiequilibrium distribution function which, for low Mach numbers, is given by $[27,28]$

$$
\begin{gathered}
f_{\alpha}^{\mathrm{eq}}(\mathbf{x}, t)=\frac{1}{9} \rho(\mathbf{x}, t)\left[1+\frac{3}{v_{\alpha}} \mathbf{v}_{\alpha} \cdot \mathbf{u}+\frac{9}{2 v_{\alpha}^{2}}\left(\mathbf{v}_{\alpha} \cdot \mathbf{u}\right)^{2}-\frac{3}{2} \mathbf{u}^{2}\right] \\
\alpha=1, \ldots, 4 \\
f_{\alpha}^{\mathrm{eq}}(\mathbf{x}, t)=\frac{1}{36} \rho(\mathbf{x}, t)\left[1+\frac{3}{v_{\alpha}} \mathbf{v}_{\alpha} \cdot \mathbf{u}+\frac{9}{2 v_{\alpha}^{2}}\left(\mathbf{v}_{\alpha} \cdot \mathbf{u}\right)^{2}-\frac{3}{2} \mathbf{u}^{2}\right] \\
\alpha=5, \ldots, 8, \\
f_{9}^{\mathrm{eq}}(\mathbf{x}, t)=\frac{4}{9} \rho(\mathbf{x}, t)\left(1-\frac{3}{2} \mathbf{u}^{2}\right) .
\end{gathered}
$$


Here, $v_{\alpha}=\left|\mathbf{v}_{\alpha}\right|$, and $\rho(\mathbf{x}, t)$ and $\mathbf{u}(\mathbf{x}, t)$ are, respectively, the density and linear macroscopic velocity of the fluid, given by

$$
\begin{gathered}
\rho(\mathbf{x}, t)=\sum_{\alpha=1}^{9} f_{\alpha}^{\mathrm{eq}}(\mathbf{x}, t)=\sum_{\alpha=1}^{9} f_{\alpha}(\mathbf{x}, t), \\
\rho(\mathbf{x}, t) \mathbf{u}(\mathbf{x}, t)=\sum_{\alpha=1}^{9} \mathbf{v}_{\alpha} f_{\alpha}^{\mathrm{eq}}(\mathbf{x}, t)=\sum_{\alpha=1}^{9} \mathbf{v}_{\alpha} f_{\alpha}(\mathbf{x}, t) .
\end{gathered}
$$

One can show [10], using a Chapman-Enskog expansion and the above equilibrium distribution function, that the continuity and Navier-Stokes equations are recovered at large times and length scales. We define the lattice spacing $\Delta x$ as the unit of length and the time step $\Delta t$ as the unit of time. The viscosity $\eta$ of the fluid is then given by, $\eta=\frac{1}{6}(2 \tau-1) \rho$ and the pressure is $P=\frac{1}{3} \rho$.

On the internal solid surface of the fractures the flow field must obey the no-slip boundary condition. The simplest way of implementing this condition is the so-called bounce-back rule in which a particle incident on the surface reverses its direction. The bounce-back rule is straightforward to implement, and is applicable to all complex solid surface geometries, but its accuracy, under certain conditions, might be limited. Inamuro, Yoshino, and Ogino [29] showed that the bounce-back rule becomes inaccurate if $\tau / \Delta t \geqslant 3$. Here, we use $\tau / \Delta t=1$, and therefore the error generated by the bounce-back rule will be very small. Other rules for implementing the no-slip boundary condition, that are supposedly more accurate than the bounce-back rule, have also been suggested [29], but they are also more complex than the bounce-back rule to implement on rough surfaces.

In our discussions below, the direction of the macroscopic flow is assumed to be from the left to right side of the square lattice (the horizontal direction of the paper), taken to be the $x$ direction. To begin the simulations, the boundary conditions must be specified, and the initial distribution $f_{\alpha}(\mathbf{x}, 0)$ for the time $t=0$ must be supplied. For this purpose, a constant pressure is applied to the network at its left side, and the fluid velocities at the entrance of all the horizontal fractures that are directly connected to this side are also assumed to be constant over their cross sections. As for $f_{\alpha}(\mathbf{x}, 0)$, we use the equilibrium distribution $f_{\alpha}^{\mathrm{eq}}$. The system then evolves according to Eqs. (5) - (8). Since the pressure is given by, $P$ $=\frac{1}{3} \rho$, it might appear that the fluid is compressible. However, the Mach number is only $\sqrt{3} \times 10^{-2}$, and therefore the fluid is only very weakly compressible.

The fluid flow through the fracture network is characterized by an effective permeability $K_{x}$. Since the fluid flow is slow, we use Darcy's law,

$$
\frac{Q}{A}=-\frac{K_{x}}{\eta} \nabla P,
$$

where $Q$ is the total volumetric flow rate leaving the network from its right side, and $A$ is the cross section area through which $Q$ passes. The volumetric flow rate can easily be computed. Since the pressure on the left side of the network is held fixed, we only need to determine an average pressure on the right side of the network. If the flow is slow enough, the effective permeability would be independent of the flow properties, and would depend only on the structural characteristics of the network, such as the roughness exponent $H$ and the maximum roughness height $h_{m}$. Thus if we define a Reynolds number Re by

$$
\operatorname{Re}=\frac{\rho(v) \ell}{\eta},
$$

where $\langle v\rangle$ is the mean fluid velocity, and $\ell$ is a characteristic length which we take it to be the average aperture $\langle\delta\rangle$ of the fracture network, then, for slow enough flows, the effective permeability is independent of Re. As described and discussed below, by varying the Reynolds number we also investigate the crossover between linear fluid flow, in which the effective permeability is independent of $\mathrm{Re}$, and the nonlinear regime.

Let us point out that, in the studies of fluid flow through consolidated or unconsolidated sandstones and similar porous media, the average or typical grain size is used as the characteristic length scale $\ell$. However, for fluid flow through the fracture networks that we study here, an average aperture, or a similar quantity, appears to be the only natural length scale for defining the Reynolds number. As such, the Reynolds numbers that we use in our study are typically smaller than those that one obtains using the average grain size as the characteristic length scale.

\section{RESULTS AND DISCUSSION}

We have carried out extensive LB simulations of fluid flow in the fracture network model described above. In what follows we describe and discuss the results.

\section{A. Characterization of the fracture network}

An important characteristic of any fracture network is the statistical distribution $F(\delta)$ of the fractures' apertures. We have constructed this distribution for two values of the roughness exponent $H$ and four values of the parameter $h_{m}$, the maximum height of the fractures' rough surfaces. Figure 2 presents the results for $H=0.1$, while the results for $H$ $=0.8$ are shown in Fig. 3. For a fixed distance between the upper and lower base (flat) surfaces of the fractures, small values of $h_{m}$ do not have much effect on the apertures of the fractures, and therefore one expects to have a narrow aperture distribution; Figs. 2 and 3 confirm this expectation. As the maximum height $h_{m}$ of the rough surfaces increases, the effect of the roughness begins to emerge, and $F(\delta)$ becomes broader. For $h_{m}=18$, the apertures vary over almost one order of magnitude. Moreover, as the roughness exponent $H$ increases, the aperture distribution becomes broader. This can be better understood by inspecting Fig. 4 which presents the variance $\sigma^{2}$ of the aperture distributions versus $H$ and $h_{m}$. For small $h_{m}$, the variance is also very small, almost independent of the roughness exponent $H$, implying that the surface roughness has little effect on any property of interest. 
(b)
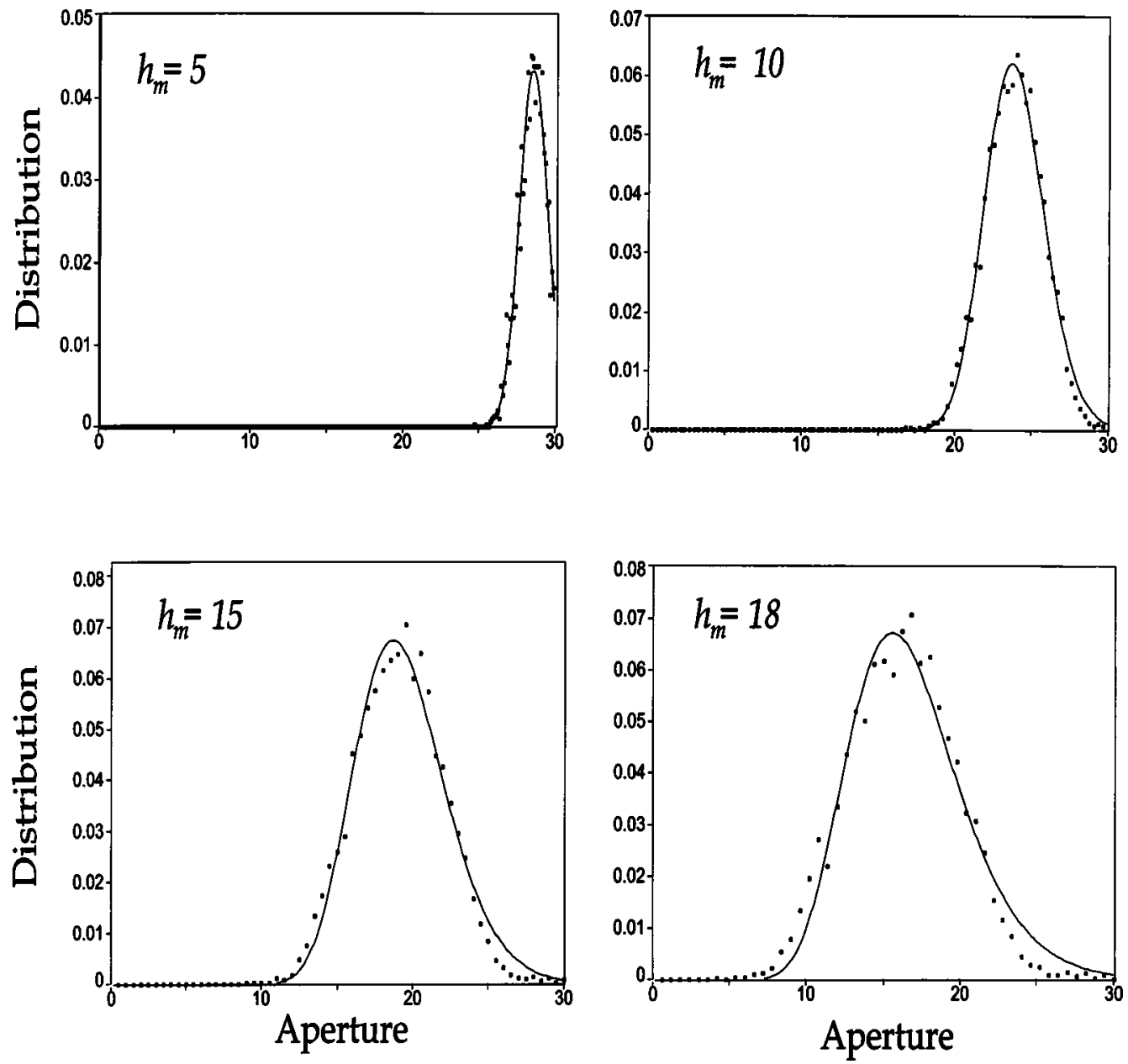

FIG. 2. Distribution of the apertures on an $L \times L$ fracture network, where $L=150$ sites, with the roughness exponent $H=0.1$. Symbols represent the numerical data, while the curves represent their numerical fits to Eq. (13).

As the fractures become narrower-(i.e., as $h_{m}$ increases), the variance increases and the effect of the surface roughness begins to manifest itself in terms of the strong dependence of $\sigma^{2}$ on the roughness exponent $H$.

Experiments by Gale [30] and others [14] have indicated that the aperture distribution of rock fracture resembles a log-normal distribution:

$$
F(\delta)=\frac{1}{\sqrt{2 \pi}(\ln 10) \sigma \delta} \exp \left\{-\frac{\left[\ln \left(\delta / \delta_{0}\right)\right]^{2}}{2 \sigma^{2}}\right\}
$$

where $\delta_{0}$ is a parameter which is related to the mean of the distribution. We have fitted the aperture distributions shown in Figs. 2 and 3 to Eq. (13); the results are also shown in these figures. As can be seen, for well-separated rough surfaces, the log-normal distribution provides excellent fits to the data. However, when the upper and lower fracture surfaces are close to each other, the fits are not as good. This is particularly true when the roughness exponent $H>\frac{1}{2}$. More experiments in which the distance between the upper and lower fracture surfaces can be controlled will shed more light on whether a log-normal aperture distribution is an accurate representation of narrow fractures (i.e., those with large $h_{m}$ ).

We should point out that a Gaussian distribution has been proposed [14] for describing the statistical properties of the fractures' internal surfaces. If this is the case, then, as described by Adler and Thovert [14], the mean aperture of any subdomain of a fracture can be fully described by two parameters, namely $\sigma_{h}^{2}$, the variance of the surface height, and $\langle s\rangle / \sigma_{h}$, where $\langle s\rangle$ is the mean separation of the upper and lower surfaces of a fracture. In addition, the size of any subdomain of the fracture has no influence on the statistical properties of the aperture distribution.

\section{B. Effective permeability of the fracture network}

Figure 5 presents a small portion of the fracture network, the magnitude of the local flow velocities, and the resulting streamlines. It is clear that the roughness of the fractures' surfaces and the associated tortuosity of their gaps generate complex flow patterns both at the fractures' junctions and inside the fractures themselves, especially in those that are aligned with the direction of the macroscopic flow field, i.e., 
(b)
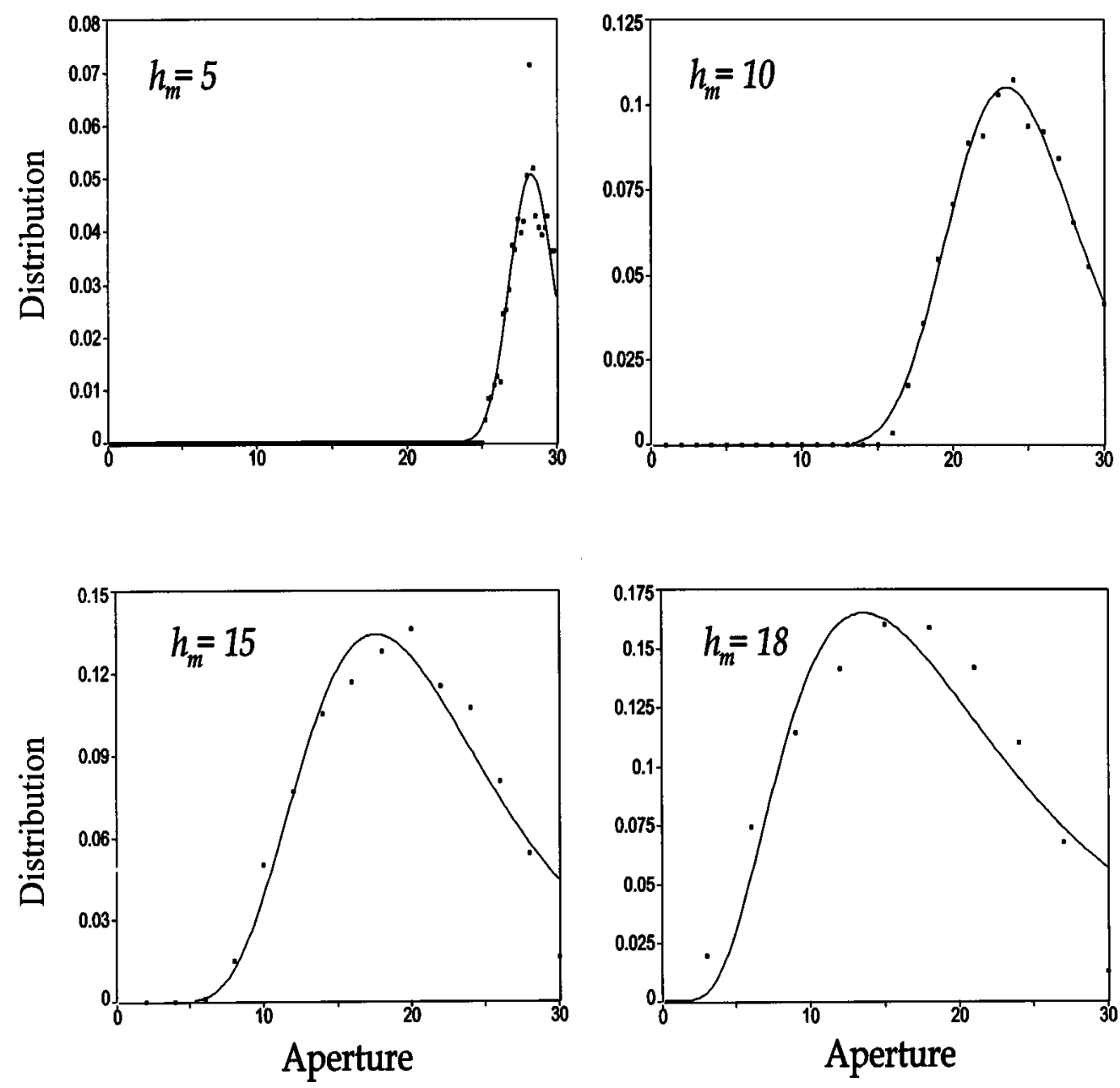

FIG. 3. Same as in Fig. 2, but with the roughness exponent $H=0.8$.

the $x$ direction. In particular, the streamlines are no longer straight, as the fluid velocity sharply decreases inside the depressions and corners. While such a pattern of the streamlines is expected, Fig. 5 is a clear demonstration of the complexities of such a pattern.

These features have an important implication for dispersion processes, i.e., unsteady mixing of two miscible fluids [1] in a flow field. If we introduce into this network tracer particles of fluid 2 to be transported (advected) by the flow field (fluid 1), then, as Fig. 5 also indicates, the tracers will follow complex paths. In particular, the traditional assumption of complete mixing of the two fluids at the fractures' junctions [1], according to which the tracers that are at the junctions choose one of the fractures there with a probability proportional to the flow rates in the fractures, is no longer valid (see, for example, Mourzenko et al. [31]). Further work on this issue is in progress; the results will be reported in a future paper [32].

Figure 6 depicts the typical variations of the effective permeability of one realization of the fracture network with the time, for $H=0.5$ and $h_{m}=10$. As this figure indicates, about 1200 time steps are typically needed for reaching a steady state. However, in percolation fracture networks, i.e., those in which some of the fractures have been removed (see below), or those in which the maximum height of fractures' surface $h_{m}>\frac{1}{2} L_{y}$, the time to achieve the steady state increases, and is in fact very large near the percolation threshold.

Figure 7 shows the dependence on the roughness exponent $H$ of the effective permeability $K_{x}$ of the fracture networks, averaged over many realizations, for a variety of fractures, ranging from narrow (large $h_{m}$ ) to wide (small $h_{m}$ ). Consistent with Figs. 2-4, the effective permeability is maximum and almost insensitive to the surface roughness when the upper and lower surfaces of the fractures are well separated (i.e., when $h_{m}$ is small). However, as the fractures become narrower (as $h_{m}$ increases), the effective permeability $K_{x}$ decreases and, moreover, exhibits strong dependence on the surface roughness. The effective permeability increases with increasing roughness exponent $H$, as larger $H$ generate surface profiles that are smoother than those with smaller $H$.

Traditionally, the effective permeability of a single fracture is written as $K \sim \delta^{m}$, where $m=2$ for fractures with completely smooth surfaces. Zhang, Knackstedt, and Sahimi 


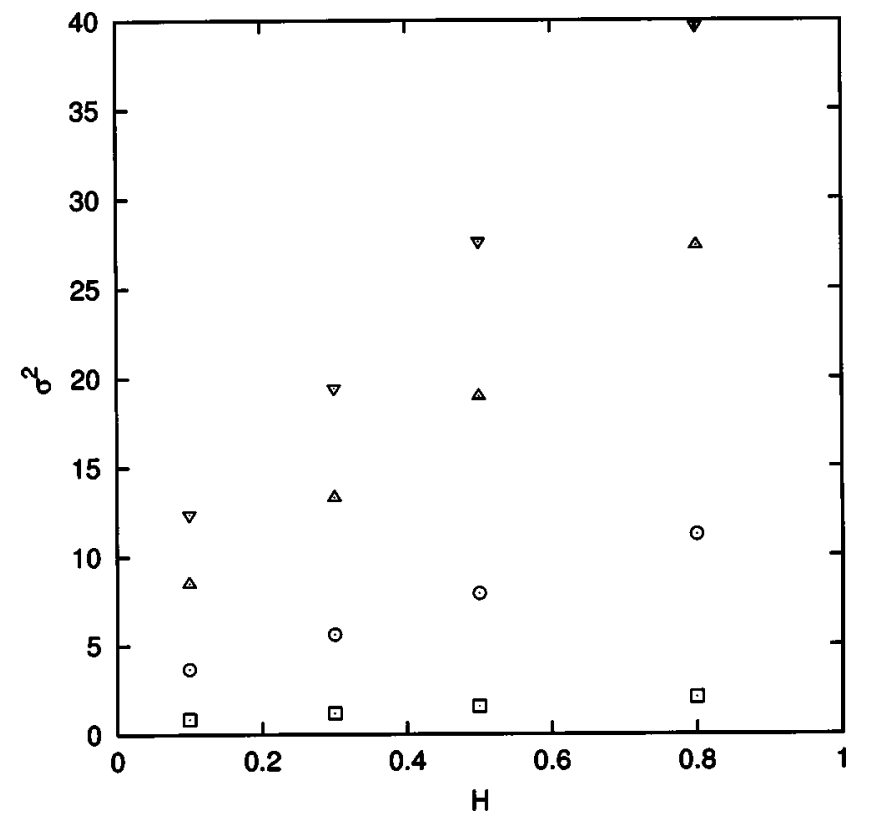

FIG. 4. Variance of the apertures versus the roughness exponent $H$, where $\square, \bigcirc, \triangle$, and $\nabla$ show, respectively, the data for $h_{m}=5$, 10,15 , and 18 .

[12] showed that with a rough, self-affine surface, one may have a $H$-dependent $m$ that can vary anywhere from 2 to 6 . We find that if we define an average aperture $\langle\delta\rangle$ for the network, then the effective permeability of the fracture network, as a function of the average aperture, appears to exhibit a well-defined power-law behavior; this is shown in Fig. 8. Thus we may write

$$
K_{x} \sim\langle\delta\rangle^{\beta} .
$$

Although it appears that the exponent $\beta$ depends on the roughness exponent $H$, due to the limited range of $\langle\delta\rangle$, we cannot at this point make any definitive statement about the

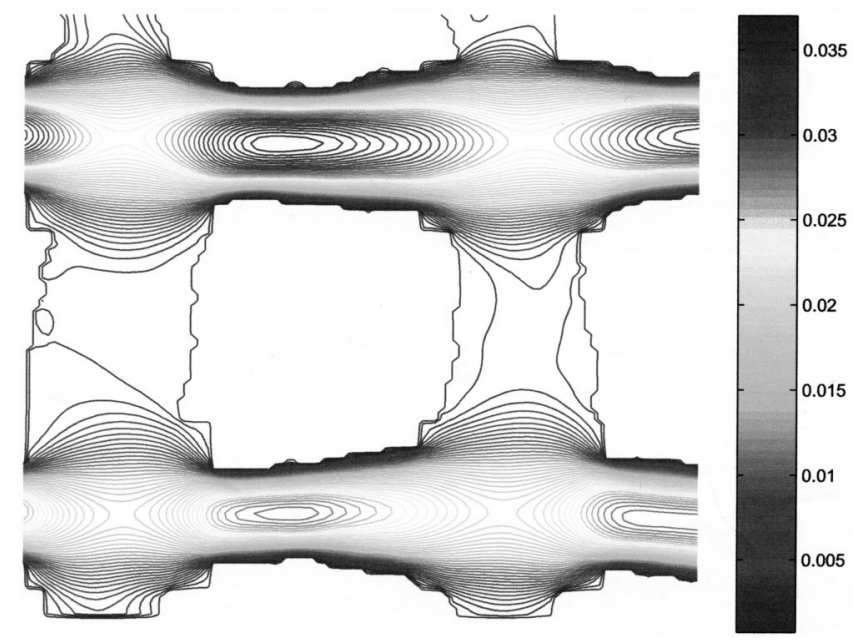

FIG. 5. The streamlines and the magnitudes of the local flow velocities in a small portion of the fracture network with the roughness exponent $H=0.5$ and $h_{m}=10$.

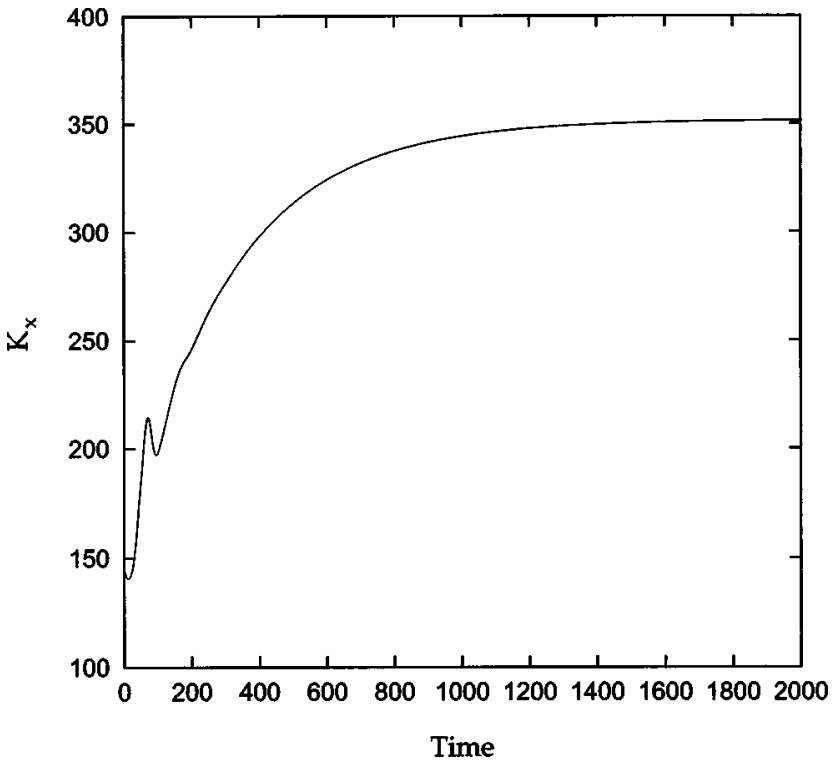

FIG. 6. The effective permeability $K_{x}$ of the fracture network versus the time, obtained with the roughness exponent $H=0.5$ and $h_{m}=10$.

function $\beta(H)$. We only observer that the effective permeability varies more rapidly with $\langle\delta\rangle$ for smoother fractures, i.e., those with a larger roughness exponent $H$. In addition, if extensive enough statistics are available, one may attempt to correlate the exponent $\beta$ with $m$, the exponent that describes the power-law dependence of the effective permeability of a single fractures on its effective aperture. In that case, the effective permeability of a fracture network would be described solely in terms of the statistics of a single fracture, which would be very useful from a practical view point.

The possible power law (14) exhibited in Fig. 8 repre-

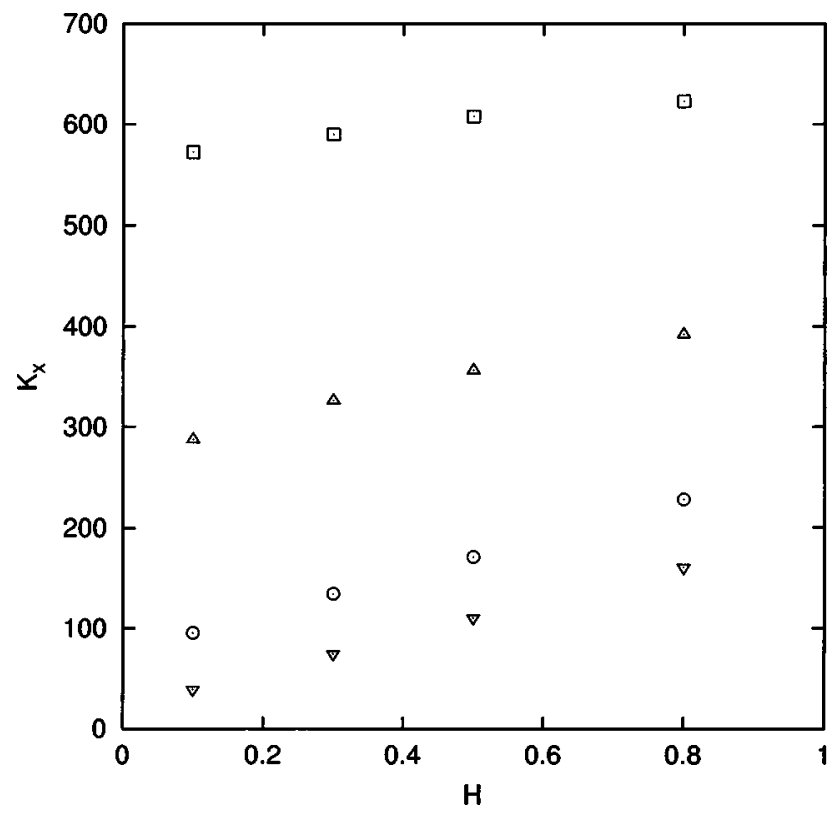

FIG. 7. The effective permeability $K_{x}$ of the fracture network as a function of the roughness exponent $H$ for $h_{m}=5(\square), 10(\triangle), 15$ $(\bigcirc)$, and $18(\nabla)$. 


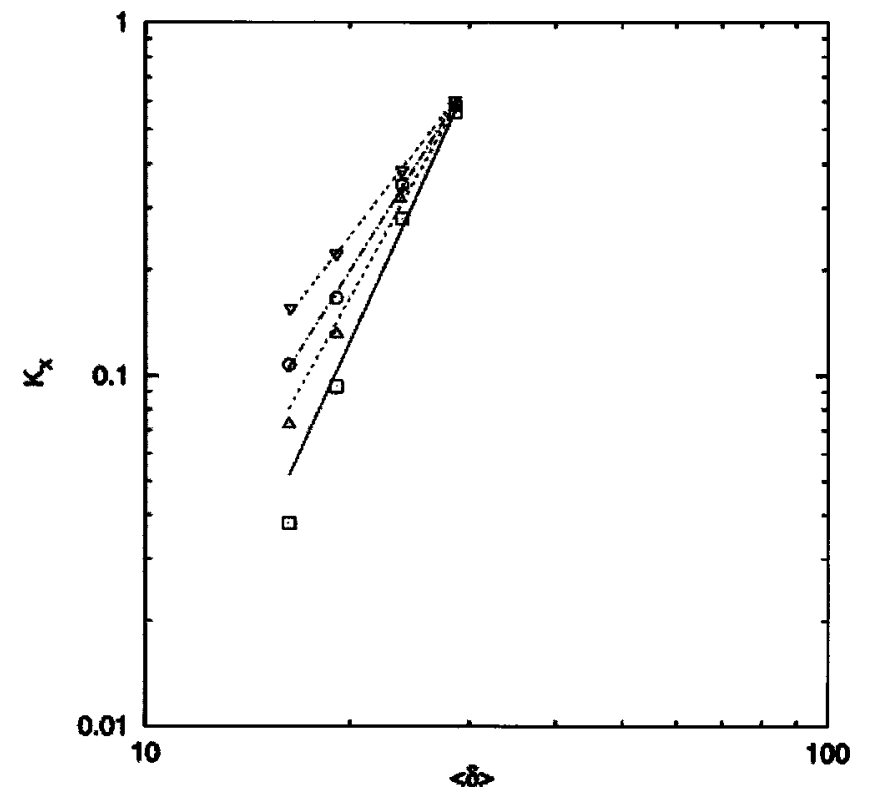

FIG. 8. Logarithmic plot of the effective permeability $K_{x}$ versus the average aperture $\langle\delta\rangle$ of the network, as a function of the roughness exponent $H$, for $H=0.1(\square), 0.3(\triangle), 0.5(\bigcirc)$, and $0.8(\nabla)$.

sents, in the following sense, an important result for simulation of flow and transport in fractured rock. In order to carry out such simulations, one first develops [1], based on the available field-scale data, a fine-grid model for the rock, usually referred to as the geological model, which typically contains millions of grid points. However, use of the geological model in the study of flow and transport in rock entails prohibitive computations involving millions of grid points and thousands of time steps, and therefore the geological model is first coarsened [33], i.e., one partitions the grid structure into relatively large cells that contain many grid points, and then represents each cell by a supergrid point. An important issue is then assigning the effective properties of the supergrid points based on the distribution of property values in their associated cells and, in particular, the effective properties of the fractures, a largely unsolved problem. Since each cell may contain a distribution of fractures, Fig. 8 indicates that the effective fracture permeability of the supergrid points (i.e., the effective fracture permeability of the fracture network inside their associated cells) is simply related to the average of the distribution of the fractures' apertures inside their associated cells through a simple power law. Full 3D simulations can determine the exponent $\beta(H)$ which can then be used in coarsening of the geological model and simulation of flow in fractured porous media.

\section{Percolating fracture networks}

As mentioned above, there is significant evidence $[1,14,15,23,24]$ indicating that the topology of fracture network of many rock formations resembles that of a percolation cluster at or very near the percolation threshold $p_{c}$. These studies analyzed the topology of the fracture networks, regardless of the magnitude of the apertures of the individual fractures, and concentrated only on the fractures' intercon-

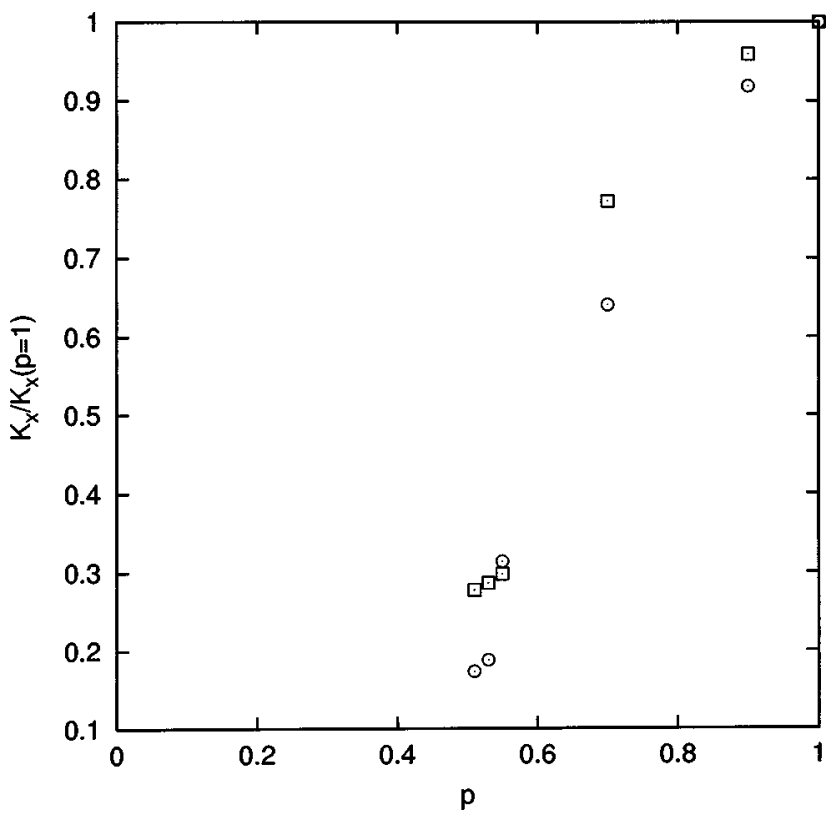

FIG. 9. The effective permeability versus the fraction $p$ of the intact fractures with the roughness exponents $H=0.3(\square)$ and 0.8 (○) and $h_{m}=10$.

nectivities. Note that, as pointed out earlier, large values of the maximum height $h_{m}$ of the fractures' rough surface does close some of the fractures, hence producing what is effectively a percolation network. However, in addition, to such networks, the results for which were described above, we also carried out extensive simulations of fluid flow in a fracture network in which only a randomly selected fraction $p$ of the fractures are intact (the rest are removed or closed), regardless of the apertures of the individual fractures. This mimics to some extent the analysis of the field-scale data for rock fracture networks $[1,14,15,23,24]$. We note that, although the structure of fracture network of rock may contain some correlations, we ignore such complexities here. As the network loses its connectivity, the time at which the flow field reaches steady state sharply increases, so that near $p_{c}$ very long simulations are necessary to reach the steady state.

Figure 9 presents the effective permeability $K_{x}(p)$ of the network, normalized by its value when the network is fully connected, for two values of the roughness exponent $H$. As expected, the effective permeability decreases with decreasing $p$. Two features of these results are note-worthy. (i) Unlike the effective conductivity of resistor networks which is typically a concave function of $p$, the effective permeability of the fracture network is a convex function of $p$, which is presumably caused by the wide distribution of the apertures. (ii) The difference between the effective permeabilities of the two networks with two different roughness exponents increases as the percolation threshold $p_{c}$ is approached, hence indicating that near $p_{c}$ the internal structure of the individual fractures plays an increasingly important role. This is evident in Fig. 10 where we show the contour plot of the magnitude of local fluid velocities in the network at $p_{c}$, after it has reached an essentially steady state, indicating an extremely complex flow pattern caused by the low connectivity of the 


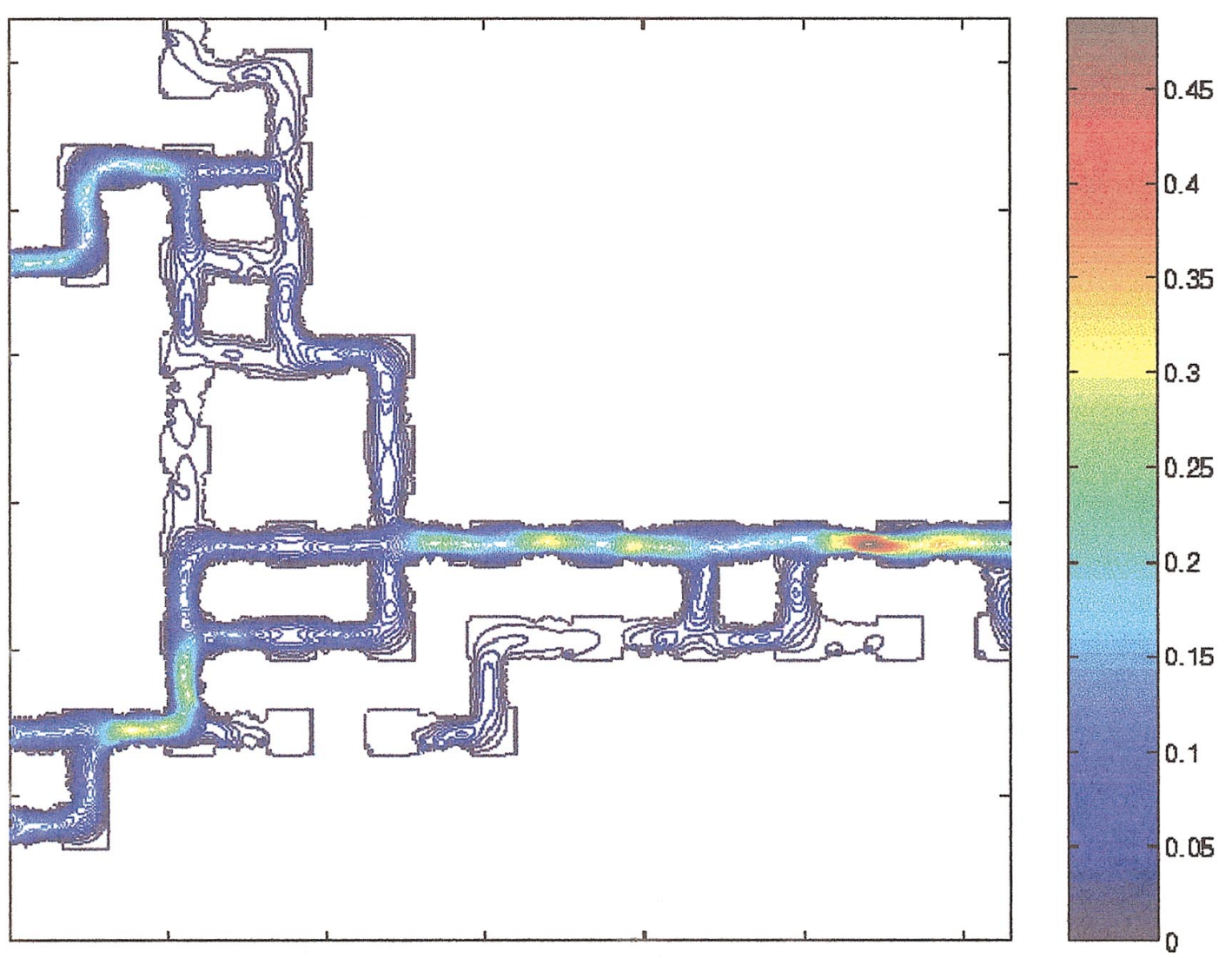

FIG. 10. (Color) The contour plot of the magnitude of the flow velocities at $p=0.5$ with $H=0.3$ and $h_{m}=10$.

network and the rough, self-affine surface of the fractures. Since near $p_{c}$ the backbone has a quasi-1D structure, the structure of the internal surface of the fractures dominates the flow pattern.

An interesting issue, at least from a theoretical view point, is whether the effective permeability of a fracture network near $p_{c}$ follows the type of power laws that one obtains for the effective conductivity of percolation networks $[16,17]$. If for $p$ near $p_{c}$ we write $K_{x} \sim\left(p-p_{c}\right)^{e}$, then since the correlation length $\xi_{p}$ of percolation is given by, $\xi_{p} \sim\left(p-p_{c}\right)^{-\nu}$, we obtain $K_{x} \sim \xi_{p}^{-e / \nu}$, while for the conductivity $g$ of resistor networks one has $g \sim \xi_{p}^{-\mu / \nu}$. In two dimensions, and with smooth internal surfaces for the fractures, one has $e=\mu$ $\simeq 1.3$. We thus computed the corresponding exponent for the fracture network with rough, self-affine surfaces. To do so, we used the standard finite-size scaling method $[16,17]$ according to which for a network of linear size $L$ at the percolation threshold $p_{c}$ one has

$$
K_{x} \sim L^{-e / \nu}
$$

To speed up the simulations, we first identified the backbone of the network, i.e., its flow-carrying part, and then carry out the LB simulations.

Typical results are shown in Fig. 11 for $H=0.3$ and $h_{m}$ $=10$. Although we could vary $L$ by only a factor of about 6 , Fig. 11 does indicate that $e \simeq \frac{2}{3}$ which, if confirmed by more extensive simulations, is very different from the exponent for the case in which the fractures have smooth surfaces. We also find that the exponent $e$ depends on the roughness exponent $H$, although, due to the very large computational times that it required, we could not obtain accurate estimates

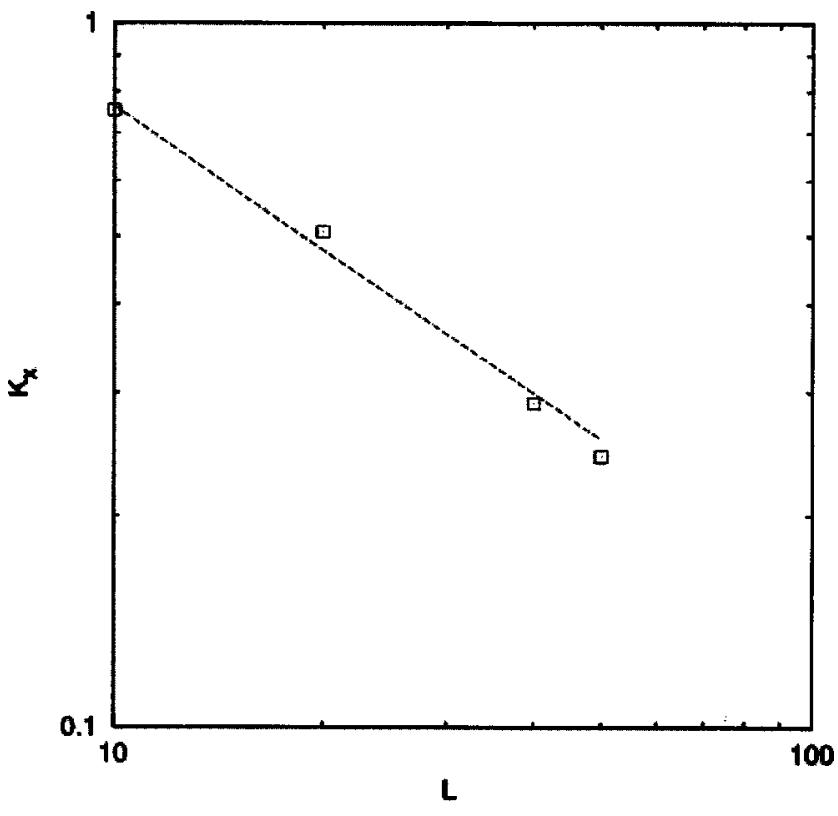

FIG. 11. Dependence of the effective permeability on the linear size $L$ of the fracture network at $p=0.5$ with $H=0.3$ and $h_{m}=10$. The dashed line represents the best fit of the data. 


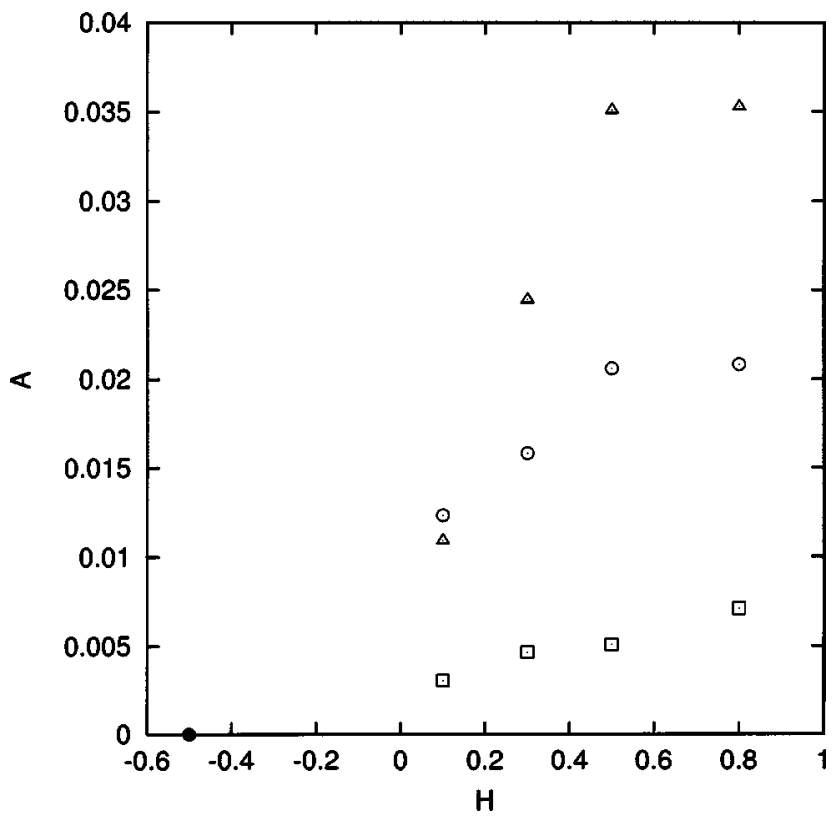

FIG. 12. Anisotropy $A$ [see Eq. (16)] for $h_{m}=5(\square), 10(\bigcirc)$, and $15(\triangle)$. The solid circle represents the anisotropy for surfaces with random roughness.

of $e$ for several other values of $H$. It would also be of interest to see whether the exponent $e$ also depends on the maximum height $h_{m}$ of the fractures' surface, at least when it is large enough that the fractures are very narrow and almost closed. More importantly, we find that for $p_{c}<p \leqslant 1$, the effective permeability $K_{x}(p)$ follows Eq. (14) with the same exponent $\beta(H)$ as for $p=1$ [the prefactor implied by Eq. (14) does depend on $p$ ], indicating the universality of $\beta$ as far as the fractures' connectivity is concerned. The implication is that, Eq. (14), if confirmed by more extensive simulations, may be used for coarsening of fracture network of rock that have incomplete or random connectivities.

\section{Finite-size effects and the anisotropy of fracture networks}

A surprising feature that we discovered in the course of our simulations is that the fracture networks that we study in this work appear to be anisotropic. That is, the effective permeabilities of the network along its two principal directions are different, although for fully connected networks the anisotropy is very small. This is an interesting, surprising, and potentially important result. A useful measure of the anisotropy of the network is the quantity $A$ defined by

$$
A=\frac{\left|K_{x}-K_{y}\right|}{K_{x}+K_{y}}
$$

so that for isotropic networks, e.g., one with smooth internal surfaces, $A=0$. Figure 12 presents the anisotropy $A$ versus the roughness exponent $H$ for three values of $h_{m}$. As can be seen, for $h_{m}=5$, when the height of the rough surface is small compared to the total height of the fracture, the anisotropy is very small. However, as $h_{m}$ increases and the fracture surface becomes rougher, the anisotropy increases.

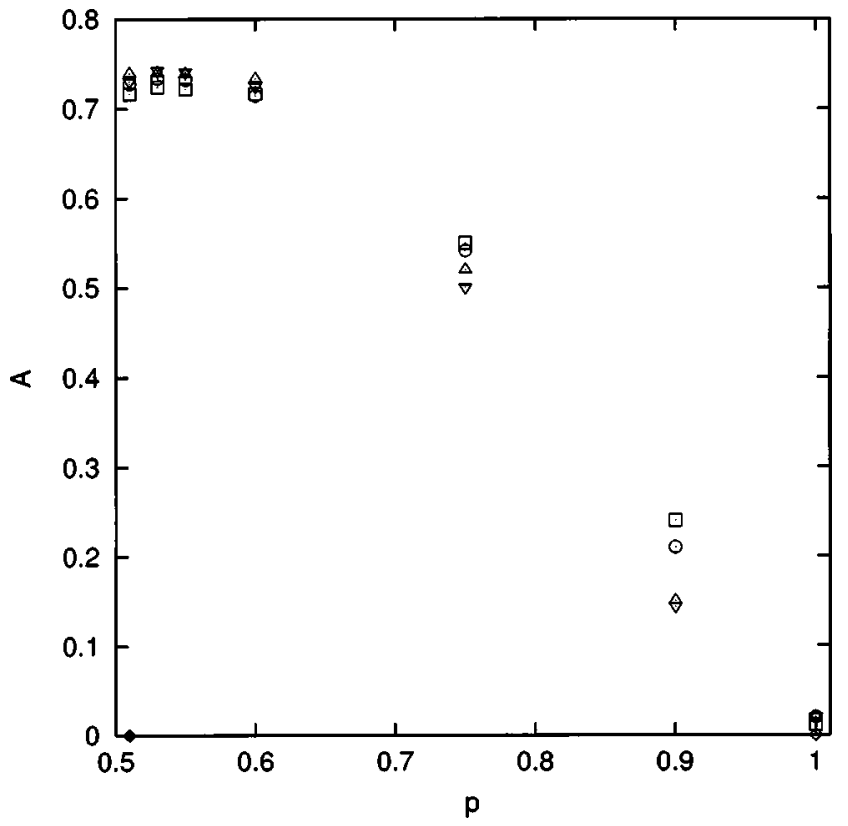

FIG. 13. Dependence of the anisotropy $A$ on $p$, the fraction of the intact fractures, with the roughness exponent $H=0.1(\square), 0.3$ $(\bigcirc), 0.5(\triangle)$, and $0.8(\nabla) .(\diamond)$ represents random roughness.

The question then is: What is the source of the anisotropy? We believe that the anisotropy is caused by the wellknown fact that the FBM is not a self-average stochastic process, implying that, if one utilizes the FBM for generating two different ensembles of values of a property (for example, the heights of the rough surfaces, as is done here), starting with two different seed numbers for the random number generations, then the statistical properties of the two ensembles will not become identical, unless their sizes are extremely large [34]. Since in constructing the fracture network, the surface roughness of each fracture is generated independently of others, the implication is that, if we construct the distributions of the fractures' apertures in the $x$ and $y$ directions, the two distributions will not be identical, even if we redistribute the fractures in the network, unless the size of the network becomes extremely large, and therefore the effective permeability of the network in the two directions will be different. Therefore, so long as the network size remains finite (even if it is large), a small will persist which will vanish only when $L$, the linear size of the network, is extremely large. Our simulations confirm this.

As a further test, we carried out a series of simulations in which the roughness of the fractures's surfaces was generated randomly, which corresponds to the limit $H=-\frac{1}{2}$ in Eq. (4), with the result being $A=0$. We then computed the anisotropy of the network as a function of $p$, the fraction of uncut fractures in the network, with both random and selfaffine rough surfaces. The results are shown in Fig. 13. While for the random roughness case the anisotropy remains zero, it increases for the case of self-affine surfaces, consistent with our argument. It is also consistent with Fig. 10 in that, as $p_{c}$ is approached, the flow field in the fracture network with self-affine surfaces is increasingly dominated by the local roughness of the fractures' surface, and therefore 


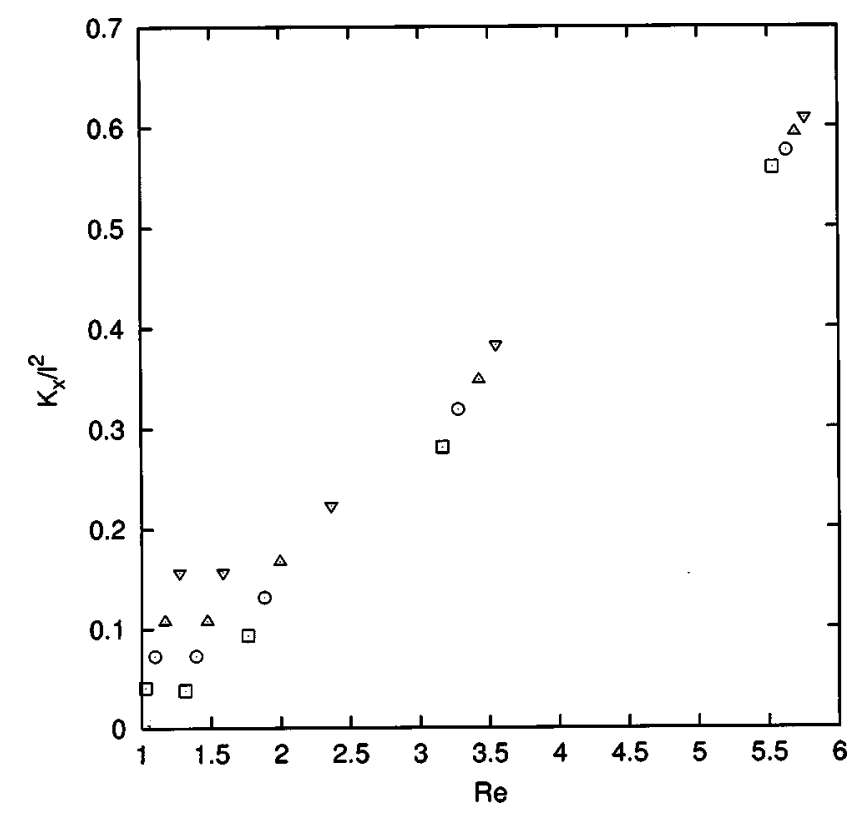

FIG. 14. Dependence of the effective permeability $K_{x}$ on the Reynolds number and the roughness exponent $H$ for $H=0.1$ ( $\square$ ), $0.3(\bigcirc), 0.5(\triangle)$, and $0.8(\nabla)$.

the anisotropy caused by such surfaces increases.

We point out that the anisotropy of the fracture networks does not imply that one has direction-dependent exponents for power laws (14) and (15). Only the prefactors in Eqs. (14) and (15) depend on the direction along which the effective permeability is measured. Our numerical computations confirm this.

\section{E. Crossover to nonlinear flow}

The last issue that we would like to take up in this paper is the crossover from a linear flow regime, in which the effective permeability of the network depends only on the microstructure of the fracture network (and is independent of the flow field) and a nonlinear one in which the apparent permeability of the network, if one still insists on using Eq. (11), would, in addition to the microstructure of the network, depend on the characteristics of the flow field, and in particular the Reynolds number.

We thus carried out a series of simulations in which the Reynolds number characterizing the flow field, and defined by Eq. (12), was varied. Figure 14 presents the dependence of the effective permeability of the fracture network on the Reynolds number for four values of the roughness exponent $H$ and $h_{m}=10$. As can be seen, for each value of $H$ there is a critical Reynolds number $\operatorname{Re}_{c}$ such that for $\operatorname{Re}<\operatorname{Re}_{c}$ the flow regime is linear, and hence the effective permeability is independent of $\mathrm{Re}$, while for $\mathrm{Re}>\mathrm{Re}_{c}$ one has a crossover to a nonlinear flow regime in which the apparent permeability of the network strongly depends on the Reynolds number. The critical Reynolds number $\operatorname{Re}_{c}$ for the crossover depends on the roughness of the surface. For rougher surfaces (smaller
$H$ ) the value of $\operatorname{Re}_{c}$ is also smaller; that is, very rough surfaces give rise to nonlinear flows even if the Reynolds is very small. The same phenomenon was observed in a single fracture with rough, self-affine surfaces [11,12]. However, we find that for a single fracture the critical Reynolds number $\operatorname{Re}_{c}$ at which the crossover occurs is larger than the corresponding value for a network of fractures. Thus we suspect that the critical Reynolds number for the crossover in a 3D fracture network would be even smaller than that of $2 \mathrm{D}$ networks considered here.

From a practical point of view, the results shown in Fig. 14 are significant. Simulation of flow of oil, gas, or groundwater in fractured natural rock is usually carried out under the assumption that the flow field is linear $[1,14]$. However, the Reynolds number in such simulations are typically as large as 5, significantly above the critical Reynolds number for the crossover to nonlinear flows.

\section{SUMMARY}

Using the lattice Boltzmann method, we have carried out extensive simulations of fluid flow in a 2D model of fracture network of rock, in which each fracture possesses rough, self-affine surfaces. The aperture distribution generated by this model is accurately represented by a log-normal distribution, which is consistent with the experimental data. The fracture network appears to be anisotropic, with the anisotropy being due to self-affinity of the fractures' surfaces, and the finite sizes of the network. The effective permeability appears to follow a power law in the mean aperture of the fracture network. A crossover from a linear to a nonlinear flow regime is obtained at a critical Reynolds number $\operatorname{Re}_{c}$ $\sim O(1)$.

In future work, we will extend the present $2 \mathrm{D}$ model to $3 \mathrm{D}$, investigate the scaling and statistical properties of the effective permeabilities of 3D fracture networks in terms of the corresponding properties at the level of a single fracture, and study dispersion and miscible displacement processes in such 3D fracture networks, phenomena that are important to enhanced recovery of oil from underground reservoirs, and to groundwater pollution.

\section{ACKNOWLEDGMENTS}

Partial support of this work by the Petroleum Research Fund, administered by the American Chemical Society, is gratefully acknowledged. This work was also supported by Ministry of Science, Research and Technology of Iran, as well as grants DFG/VE 163/4-1.5 (project "Forschergruppe: Modellierung kohäsiver Reibungsmaterialien als Kontinuum oder Diskontium, Teilprojeck V") and SFB 381 (project "Charakterizierung des schädingungsverlauf in Faserverbundwerkstoffen mittels zerstjrungsfreier Prüfung, Teilprojeck C7"). M.M. is grateful to Hans Herrmann and Stefan Luding for warm hospitality at the University of Stuttgart, where most of this work was carried out. 
[1] M. Sahimi, Rev. Mod. Phys. 65, 1393 (1993); Flow and Transport in Porous Media and Fractured Rock (VCH, Weinheim, Germany, 1995).

[2] S. R. Brown and C. H. Scholz, J. Geophys. Res. B 90, 12575 (1985).

[3] S. R. Brown, Geophys. Res. Lett. 14, 1095 (1987); J. Geophys. Res. B 92, 1337 (1987).

[4] J. Schmittbuhl, S. Gentier, and S. Roux, Geophys. Res. Lett. 20, 639 (1993).

[5] B. L. Cox and J. S. Y. Wang, Fractals 1, 87 (1993); N. E. Odling, Rock Mech. Rock Eng. 27, 13 (1994).

[6] D. T. Snow, Int. J. Rock Mech. Min. Sci. Geomech. Abstr. 7, 23 (1970); R. A. Vickers, S. P. Neuman, M. J. Sully, and D. D. Evans, Geophys. Res. Lett. 19, 1029 (1992); R. A. Johns, J. S. Steuds, L. M. Castainer, and P. V. Roberts, J. Geophys. Res. B 98, 1889 (1993).

[7] M. Sahimi, Heterogeneous Materials; Volume II: Nonlinear and Breakdown Properties, and Atomistic Modelling (Springer, New York, 2003); Phys. Rep. 306, 213 (1998).

[8] Y. W. Tsang and P. A. Witherspoon, J. Geophys. Res. 86, 9287 (1981); E. Hakami and E. Larsson, Int. J. Rock Mech. Min. Sci. Geomech. Abstr. 33, 395 (1996).

[9] V. M. Mourzenko, J.-F. Thovert, and P. M. Adler, J. Phys. II 5, 465 (1995).

[10] D. H. Rothman and S. Zaleski, Lattice-Gas Cellular Automata (Cambridge University Press, London, 1997).

[11] R. Gutfraind and A. Hansen, Transp. Porous Media 18, 131 (1995)

[12] X. Zhang, M. A. Knackstedt, and M. Sahimi, Physica A 233, 835 (1996).

[13] G. Drazer and J. Koplik, Phys. Rev. E 62, 8076 (2000); C. DeW. Van Siclen, J. Geophys. Res. B 107, 2106 (2002); M. Madadi, C. DeW. Van Siclen, and M. Sahimi, J. Geophys. Res. (to be published).

[14] P. M. Adler and J.-F. Thovert, Fractures and Fracture Networks (Kluwer, Boston, 1999).

[15] T. Chelidze and Y. Gueguen, Int. J. Rock Mech. Min. Sci. Geomech. Abstr. 27, 223 (1990).

[16] D. Stauffer and A. Aharony, Introduction to Percolation Theory, 2nd ed. (Taylor and Francis, London, 1992); Fractals and Disordered Systems, 2nd ed., edited by A. Bunde and S. Havlin (Springer, Berlin, 1996).

[17] M. Sahimi, Applications of Percolation Theory (Taylor and Francis, London, 1994).

[18] R. Englman, Y. Gur, and Z. Jaeger, J. Appl. Mech. 50, 707 (1983).

[19] P. C. Robinson, J. Phys. A 17, 2823 (1984).

[20] K. Hestir and J. C. S. Long, J. Geophys. Res. B 95, 21565 (1990).

[21] I. Balberg, B. Berkowitz, and G. E. Drachsler, J. Geophys. Res. B 96, 10015 (1991).

[22] S. Mukhopadhyay and M. Sahimi, in Proceedings of the Society of Petroleum Engineers Western Regional Meeting, Paper SPE 24043, Bakersfield, CA (Society of Petroleum Engineers, Richardson, TX, 1992).

[23] B. Berkowitz, Math. Geol. 27, 467 (1995).

[24] O. Bour and P. Davy, Water Resour. Res. 33, 1567 (1997); 34, 2611 (1998).

[25] N. Koudina, R. Gonzales Garcia, J.-F. Thovert, and P. M. Adler, Phys. Rev. E 57, 4466 (1998).

[26] V. V. Mourzenko, J.-F. Thovert, and P. M. Adler, Phys. Rev. E 59, 4265 (1999); Transp. Porous Media 45, 89 (2001).

[27] Q. Zau, S. Hou, S. Chen, and G. D. Doolen, J. Stat. Phys. 81, 35 (1995).

[28] X. He and L.-S. Luo, Phys. Rev. E 56, 6811 (1997).

[29] T. Inamuro, M. Yoshino, and F. Ogino, Phys. Fluids 7, 2928 (1995); L. Ginzbourg and P. M. Adler, J. Phys. II 4, 191 (1994).

[30] J. E. Gale, in Proceedings of the 28th U.S. Symposium on Rock Mechanics, Tucson, AZ 1987 (Balkema, Rotterdam, 1988), p. 1213.

[31] V. V. Mourzenko, F. Yousefian, B. Kolbah, J.-F. Thovert, and P. M. Adler, Water Resour. Res. 38, 10.1029/2000WR000211 (2002).

[32] M. Madadi and M. Sahimi (unpublished).

[33] A. R. Mehrabi and M. Sahimi, Phys. Rev. Lett. 79, 4385 (1997); F. Ebrahimi and M. Sahimi, Physica A 316, 160 (2002), and references therein.

[34] M. B. Isichenko, Rev. Mod. Phys. 64, 961 (1992); C. Du, C. Satik, and Y. C. Yortsos, AIChE J. 42, 2392 (1996); M. Sahimi and S. Mukhopadhyay, Phys. Rev. E 54, 3870 (1996). 